\title{
Competencia digital, actitudes y expectativas hacia las tecnologías digitales. Perfil de los futuros maestros de primaria
}

\author{
Digital competence, attitudes and expectations towards digital \\ technologies. Profile of future elementary teacher
}

\author{
María Ángeles Llopis Nebot \\ Universitat Jaume I de Castellón (España) \\ mallopis@uji.es \\ María Santágueda Villanueva \\ Universitat Jaume I de Castellón (España) \\ santague@uji.es \\ Francesc Marc Esteve Mon \\ Universitat Jaume I de Castellón (España) \\ festeve@uii.es
}

Recibido: $24 / 02 / 2021$

Aceptado: $12 / 09 / 2021$

Publicado: 01/12/2021

\section{RESUMEN}

Este estudio pretende trazar un perfil del alumnado que cursa el Grado de Maestro en Educación Primaria en relación a sus hábitos en el uso de las tecnologías digitales, la percepción sobre su competencia digital, así como de las expectativas y actitudes hacia el uso de la tecnología en su ámbito personal y académico. Se realiza asimismo una comparación entre alumnos pertenecientes a dos planes de estudios distintos. Los datos se obtienen mediante el cuestionario INCOTIC 2.0. Tras el análisis de datos se evidencia el alto uso que hacen de la tecnología a través de dispositivos móviles y portátiles, especialmente en el ámbito de las relaciones sociales. Se muestra una buena actitud hacia el uso académico de la tecnología y una favorable expectativa de uso. En cuanto a la percepción de la competencia digital, destacan en las alfabetizaciones comunicativa y multimedia. Comparando ambos grupos, no se observan diferencias significativas en la mejora de la competencia digital, estas se limitan al ámbito comunicativo, las actitudes y las expectativas que tiene el estudiantado hacia las TIC. De acuerdo con los resultados, se propone incidir en la mejora de las áreas informacional y tecnológica, ambas fundamentales para su desarrollo profesional docente.

\section{PALABRAS CLAVE}

Competencia digital; educación superior; tecnologías digitales; formación inicial docente.

\begin{abstract}
This study aims to draw a profile of the students who are studying the Bachelor's Degree in Primary Education in relation to their habits in the use of digital technologies, the perception of their digital competence, as well as the expectations and attitudes towards the use of technology in their personal and academic environment. A comparison is also made between students belonging to two different study plans. The data is obtained through the INCOTIC 2.0 questionnaire. After data analysis, the high use they make of technology through mobile and portable devices is evident, especially in the field of social relationships. In addition, there is a good attitude towards the academic use of technology and a favorable expectation of use. Regarding the perception of digital competence, they stand out in communicative and multimedia literacies. However, in the comparison of both groups, no significant differences are observed in the improvement of digital competence, and these are limited to the communicative field of these
\end{abstract}


technologies, attitudes and expectations that the students have towards digital technologies. According to the results, it is proposed to influence the improvement of the informational and technological areas, both vital for their teaching professional development.

\section{KEYWORDS}

Digital competence; higher education; digital technologies; initial teacher training.

\section{CITA RECOMENDADA}

Llopis, M.Á., Santágueda, M. \& Esteve, F.M. (2021). Competencia digital, actitudes y expectativas hacia las tecnologías digitales. Perfil de los futuros maestros de primaria. RIITE Revista Interuniversitaria de Investigación en Tecnología Educativa, 11, 114-130. https://doi.org/10.6018/riite.470331

\section{Principales aportaciones del artículo y futuras líneas de investigación:}

- Trazar un perfil del estudiantado universitario actual en cuanto al uso y disponibilidad de las tecnologías digitales.

- Analizar la competencia digital de dicho estudiantado con el fin de conocer las áreas más favorables y las más deficientes.

- Averiguar si existen diferencias generacionales significativas en la competencia digital del estudiantado del Grado de Maestro en Educación Primaria de dos planes de estudios distintos.

- Líneas futuras: estudiar si se dan cambios en los resultados obtenidos a partir de la situación de confinamiento que se generó por la pandemia de la COVID-19.

\section{INTRODUCCIÓN}

La adopción y el uso de las tecnologías digitales ha experimentado un crecimiento significativo en las últimas décadas, sobre todo entre la población más joven (Bullen et al., 2011), quienes utilizan habitualmente todo tipo de tecnologías, especialmente dispositivos móviles y tecnologías sociales y audiovisuales.

A pesar de la expansión del uso de las tecnologías digitales (TD) y que, por ende, pudiera parecer que los jóvenes poseen altas habilidades en su manejo, resulta necesario incidir en la importancia de desarrollar una adecuada competencia digital en las diferentes etapas formativas (Comisión Europea, 2018).

Esta competencia resulta indispensable para hacer un uso adecuado de la tecnología, y su relevancia, se ha evidenciado aún más, tras la situación de emergencia sanitaria derivada por la pandemia del COVID-19 (Vogels et al., 2020), especialmente en el ámbito educativo. La competencia digital resulta necesaria en la formación de estudiantes universitarios de los grados de educación, es decir, futuros maestros (Prendes et al., 2010), población en la que se centra esta investigación.

El propósito del presente estudio es profundizar en el perfil digital de los estudiantes universitarios actuales y en particular en el Estudiantado del Grado de Maestro en Educación Primaria, a partir del análisis de sus usos, preferencias, habilidades y expectativas hacia las TD. En concreto, el artículo pretende dar respuesta a las siguientes preguntas de investigación:

1. ¿Cuáles son las características actuales de los estudiantes universitarios en cuanto a la disponibilidad y al uso de las TD?

2. ¿Son competentes digitalmente los estudiantes universitarios? 
3. ¿Cuáles son las actitudes y expectativas de los estudiantes universitarios hacia el uso de las TD?

4. ¿Existen diferencias generacionales en cuanto al perfil digital del estudiantado universitario?

\subsection{El perfil digital del estudiantado universitario actual}

Internet se ha convertido en el medio más habitual de información de los jóvenes, sustituyendo a la televisión; estos se informan principalmente a través de la red, ya sea de forma directa a través de webs y aplicaciones de prensa, como de manera indirecta a través de redes sociales (Antunovic, et al., 2018). El hogar sigue siendo el lugar más común para que adolescentes y niños accedan a Internet, especialmente a través de dispositivos móviles (Livingstone, et al., 2019).

En este sentido, niños y jóvenes comienzan a utilizar teléfonos móviles a edades cada vez más tempranas, debido a las ventajas que suponen, al ser una herramienta de carácter personal, portátil y de fácil uso por las interfaces táctiles (Goggin, 2013). A edades tempranas, el uso de teléfonos inteligentes se utiliza princiapalmente tanto para acceder a juegos y vídeos, como para satisfacer necesidades sociales (Stoilova et al., 2020). Asimismo, los móviles, por sus posibilidades tecnológicas y su valor educativo, son un elemento que está cada vez más integrado en las instituciones educativas (Camacho \& Esteve, 2018).

Aunque a menudo los jóvenes se sienten abrumados por las redes sociales y la presión de construir una imagen o una identidad digital positiva, también atribuyen a estas plataformas valores beneficiosos, como son el fortalecimiento de amistades, la exposición a opiniones diversas, y la posibilidad de apoyar causas que les preocupan (Boyd, 2014). Según Anderson y Jiang (2018), aproximadamente ocho de cada diez jóvenes, afirman que las redes sociales les hacen sentir más conectados con sus amistades, mientras que alrededor de dos tercios explican que utilizar estos medios les permite sentirse apoyados en momentos difíciles.

Este uso masivo de la tecnología entre la población más joven ha hecho que se popularicen ciertos mitos sobre la existencia de una nueva y supuesta generación de jóvenes digitales (Gallardo-Echenique et al., 2015; Hatlevik \& Christophersen, 2013). Durante la primera década de este siglo, diferentes autores afirmaron que las características de los estudiantes actuales habían cambiado radicalmente respecto a los de décadas anteriores, denominando a estas generaciones como nativos digitales (Prensky, 2001), Generación Net (Oblinger \& Oblinger, 2005) o aprendices del nuevo milenio (Pedró, 2009). Sin embargo, Selwyn (2009) y Calvani et al. (2012) no encuentran suficientes evidencias para afirmar la existencia de dos generaciones separadas, sino más bien hallan una alta heterogeneidad entre estudiantes. Otros autores (Claro et al., 2012; Kennedy et al., 2007; Valtonen et al., 2011) observan que, aunque ciertas capacidades digitales se encuentran muy desarrolladas entre los jóvenes, estas son habilidades tecnológicas ligadas a actividades sociales o lúdicas, y a menudo no son capaces de transferirlas a otros contextos, como pudiera ser el educativo, consiguiendo favorecer su aprendizaje.

\subsection{De la alfabetización a la competencia digital}

Uno de los primeros y más utilizados términos para referirse a la habilidad para utilizar adecuadamente las tecnologías digitales es la alfabetización digital. Acuñado por Gilster (1997), se define como la capacidad de comprender y usar información, en múltiples formatos y fuentes, cuando esta se presenta a través de ordenadores. Otros autores, como Martin (2005), Bawden (2008) o Covello (2010) completan esta definición, describiendo una serie de habilidades y actitudes, como (1) identificar la necesidad de información; (2) acceder, buscar y recuperar la información a través de las tecnologías; (3) organizar y clasificar dicha información a través de las TIC; (4) leerla, entenderla e integrarla, en múltiples formatos y materiales dinámicos; (5) adaptar la información y crear mediante medios digitales; y (6) comunicar y transmitir a través de las tecnologías de la información y la comunicación (TIC), considerando los diferentes medios y canales digitales. Según las conceptualizaciones anteriores, la alfabetización digital se entiende como el conjunto de habilidades y destrezas en relación a las 
TIC, y se considera una competencia básica o clave para el siglo XXI (Solomon et al., 2011), siendo esta una concepción instrumental de la tecnología (Spante et al., 2018).

En paralelo al término alfabetización digital surgen múltiples vocablos relacionados, como alfabetización TIC, o habilidades digitales e informáticas, que suelen usarse de manera intercambiable, por su similar significado (Hatlevik \& Christophersen, 2013; Siddiq \& Scherer, 2019). Si bien el término alfabetización digital suele ser el más empleado a nivel internacional, especialmente en el contexto europeo, donde se utiliza de forma sinónima al de competencia digital (Calvani et al., 2012).

Sin embargo, alfabetización y competencia son vocablos que no siempre tienen las mismas connotaciones, y en el uso de uno u otro influye tanto el contexto lingüístico como el marco conceptual del que se parte (Meyers et al., 2013). Según Ferrari et al. (2012), existen dos principales aproximaciones al concepto de alfabetización o competencia digital. Desde el primer enfoque, se entiende la competencia digital como la convergencia de múltiples alfabetizaciones, mientras que desde la otra aproximación, la competencia digital resulta por sí misma una nueva alfabetización.

A partir del análisis de diferentes marcos, Larraz (2012) define la competencia digital como la suma de cuatro alfabetizaciones: (1) informacional, gestión de la información digital; (2) tecnológica, tratamiento de datos en diferentes formatos; (3) multimedia, análisis y creación de mensajes multimedia; y (4) comunicativa, participar de manera segura, ética y cívica desde una identidad digital. Según el Joint Research Centre de la Comisión Europea (Ferrari, 2013) la competencia digital se compone de 21 competencias agrupadas en 5 áreas: (1) información, identificar, localizar, recuperar, almacenar, organizar y analizar la información digital; (2) comunicación, interactuar en entornos digitales, compartir recursos, conectar y colaborar, y participar en redes; (3) creación de contenidos, crear y editar contenidos multimedia nuevos, integrar y reelaborar, programar y aplicar los derechos de propiedad intelectual; (4) seguridad, protección personal, de datos, de identidad digital, uso seguro y sostenible; y (5) resolución de problemas, identificar necesidades y recursos digitales, tomar decisiones, elegir las herramientas adecuadas, resolver problemas conceptuales y técnicos, y actualizar la propia competencia y la de otros.

Más recientemente, la Comisión Europea (2018), define la competencia digital como la suma de la alfabetización en información y datos, la comunicación y la colaboración, la alfabetización mediática, la creación de contenidos digitales, la seguridad, la propiedad intelectual, la resolución de problemas y el pensamiento crítico. En este sentido, las personas, además de saber reconocer e interactuar efectivamente con el software y los diferentes dispositivos, deben ser capaces de gestionar y proteger la información, los contenidos, los datos y la identidad digital. Se trata de una competencia que implica el uso seguro, crítico y responsable de las tecnologías digitales para el aprendizaje, en el trabajo y para la participación en la sociedad. Es, por lo tanto, una de las competencias fundamentales para el desempeño de la profesión docente (Caena \& Redecker, 2019; Prendes et al., 2010).

\subsection{Actitudes hacia las TD y expectativas de uso}

Las escuelas disponen hoy en día de mejor conexión a internet, más ordenadores por número de estudiantes y en general, mayor cantidad de herramientas tecnológicas útiles para la docencia. También se ha incrementado la cantidad y calidad de la formación docente en el ámbito de las TD. Sin embargo, este incremento de recursos no se ha traducido en un aumento proporcional de su uso en el aula ni en un cambio metodológico notable (Ala-Mutka, 2011).

Domingo y Marqués (2011), identifican que las herramientas TIC más frecuentemente utilizadas en las escuelas son los ordenadores y las pizarras digitales, utilizadas principalmente para apoyar la metodología en uso, pero no tanto para la innovación de esta.

Los condicionantes de la introducción y uso de la tecnología en el aula están relacionados por un lado, con características internas del docente, como su conocimiento tecnológico, el potencial pedagógico que atribuye a una determinada tecnología, la actitud hacia las TIC o la apertura hacia la innovación (Windschitl \& Sahl, 2002). Otros condicionantes no dependen 
tanto del propio docente sino del contexto educativo que le rodea, como aspectos organizativos del centro o la cultura pedagógica de este (Tejedor \& García-Valcárcel, 2006).

Uno de los modelos más utilizados para explicar cómo la tecnología es implementada con éxito en un determinado contexto es el Modelo de Aceptación Tecnológica (TAM) de Davis (1989). Este modelo, en su tercera versión o TAM3 (Venkatesh \& Bala, 2008), explica el proceso de aceptación tecnológica en base a dos constructos motivacionales extrínsecos. Por un lado, la utilidad percibida, es decir, el grado en el que una persona cree que utilizar una determinada tecnología mejorará su vida personal o profesional, y por otro, la sencillez de uso percibida o grado en el que cree que utilizar una determinada tecnología no supondrá demasiado esfuerzo. EI TAM3 aporta como factores moduladores de la utilizad y la sencillez percibida, elementos intrínsecos como el disfrute, la resistencia al cambio o la experiencia previa en el uso de la tecnología.

Karahanna et al. (2006), revisan la Teoría de la Difusión de Innovaciones (IDT) de Rogers (1962) y sugieren que la decisión de utilizar una determinada tecnología en un contexto específico, está relacionada con la compatibilidad percibida entre dicha tecnología y los valores personales, el método de trabajo y la experiencia previa.

En el campo educativo, para aquellos maestros en formación, uno de los aspectos que parecen ser determinantes para la adopción tecnológica y el cambio metodológico es la experiencia previa. Según Oreg (2003), las vivencias de los estudiantes durante sus primeros ciclos educativos así como la experiencia extraída a partir de la observación de otros maestros en activo, determinan la probabilidad de utilizar metodologías de aula que incluyan las TD. Guo et al. (2013), encuentran que si estas vivencias previas no ocurren, la introducción de tecnología en el aula cuando se incorporen como profesionales, generará mayor resistencia.

\subsection{Objetivo y preguntas de investigación}

El propósito del presente estudio es profundizar en el perfil digital de los estudiantes universitarios actuales y en particular en el Estudiantado del Grado de Maestro en Educación Primaria, a partir del análisis de sus usos, preferencias, habilidades y expectativas hacia las TD, así como profundizar en las posibles diferencias generacionales entre los estudiantes según los diferentes planes de estudio.

En concreto, el artículo pretende dar respuesta a las siguientes preguntas de investigación:

P.1. ¿Cuáles son las características actuales de los estudiantes universitarios en cuanto a la disponibilidad y al uso de las TD?

P.2. ¿Son competentes digitalmente los estudiantes universitarios?

P.3. ¿Cuáles son las actitudes y expectativas de los estudiantes universitarios hacia el uso de las TD?

P.4. ¿Existen diferencias generacionales en cuanto al perfil digital del estudiantado universitario?

\section{MÉTODO}

\subsection{DISEÑO DE LA INVESTIGACIÓN}

La presente investigación se basa en un análisis exploratorio de datos, un enfoque cuantitativo que, partiendo del análisis descriptivo, pretende relevar posibles patrones haciendo uso de diferentes análisis (Little, 2013). En concreto, en esta investigación se utilizaron principalmente técnicas descriptivas e inferenciales a partir de los resultados de un cuestionario, como a continuación se describe. 


\subsection{Contexto}

El estudio se ha llevado a cabo en una universidad española de tamaño medio, que cuenta con un total aproximado de 14.000 estudiantes, de los cuales, cerca de 600 son estudiantes del Grado en Maestro/a de Educación Primaria.

La recogida de datos tuvo lugar en dos periodos temporales diferentes, el primero de ellos durante el curso 2016-2017 (Plan de 2010) y el segundo durante el curso 2019-2020 (Plan de 2018). Dichos periodos dieron lugar a, por un lado, un relevo generacional en el estudiantado, es decir, entre la primera recogida de datos y la segunda, pasaron cuatro años, pudiendo haberse dado un cambio de hábitos. Por otro lado, cada uno de los grupos analizados formó parte de un plan de estudios diferente, siendo característica diferenciadora entre el primer plan y el segundo, que este último apostó por una intensificación de la formación práctica, iniciada desde los primeros cursos, frente al alumnado del Plan de 2010, que no tuvo dicha intensificación.

\subsection{Participantes}

En total han participado 296 estudiantes, de $2^{\circ}$ curso de dicho grado, el $49 \%$ pertenecientes al plan de estudios de 2010, y el 51\% pertenecientes al plan de 2018 (tabla 1). El porcentaje de hombres que participaron fue de $34.12 \%$ y el de mujeres de $65,88 \%$, con edades comprendidas entre los 18 y los 42 años $(M=20,3$, y $S D=2,6)$.

Tabla 1. Muestra final de los participantes $(N=296)$

\begin{tabular}{lccc}
\hline & & Frecuencia $(\mathrm{n})$ & Porcentaje $(\%)$ \\
\hline Género & Mujeres & 195 & 65,9 \\
& Hombres & 101 & 34,1 \\
Edad & $18-22$ & 257 & 86,8 \\
& +22 & 39 & 13,2 \\
Plan de & Plan 2010 & 144 & 48,6 \\
estudios & & & \\
& Plan 2018 & 152 & 51,4 \\
\hline
\end{tabular}

\subsection{Instrumento}

Para llegar a cabo la investigación se ha utilizado el cuestionario de autopercepción de competencia digital, INCOTIC 2.0, creado por González et al. (2018), que cuenta con un total de 104 ítems, distribuidos en las siguientes secciones: (1) Contextualización del instrumento e información del estudio; (2) Biodatos; (3) Disponibilidad de recursos TIC; (4) Uso de las TIC. Frecuencia general de uso de dispositivos y aplicaciones, y finalidad. (5) Autopercepción de la competencia digital, dividida en las alfabetizaciones informacional, tecnológica, multimedia y comunicativa; (6) Actitudes y expectativas hacia las TIC.

Si bien la primera sección cuenta con ítems dicotómicos, el resto de los ítems del cuestionario siguen una escala de tipo Likert del 1 al 5 , siendo 1 Nunca/Nada, y 5 Siempre/Mucho. Se trata de un instrumento ya validado previamente por los autores (González et al., 2018), y que cuenta con un resultado de fiabilidad excelente según el Alfa de Cronbach (0.91).

\subsection{Procedimiento}

La recogida de datos se llevó a cabo en dos periodos. El primero de ellos, con el alumnado de segundo curso, matriculado en la asignatura Nuevas Tecnologías Aplicadas a la Educación, ubicada en el segundo semestre académico, durante el curso 2016-2017 (Plan de 2010) y el segundo periodo, con el alumnado equivalente de la misma asignatura cursada en 2019-2020 (Plan de 2018). El cuestionario INCOTIC 2.0 se aplicó vinculado al bloque temático de competencia digital docente, siendo de obligada respuesta para todo el alumnado. Se respondió en el aula, de manera telemática, dejando en ambos casos 20 minutos para ello. 


\subsection{Análisis de los datos}

Para realizar el análisis de datos se utilizó el programa IBM SPSS Statistics Versión 25. En primer lugar se calcularon los resultados descriptivos de los diferentes ítems y secciones 0 dimensiones. Seguidamente se realizó la prueba no paramétrica $U$ de Mann-Whitney para comparar las medias entre los estudiantes de los dos planes de estudios.

En la tabla 2 se puede observar la relación entre las preguntas de investigación y la prueba estadística aplicada. Para las tres primeras preguntas, disponibilidad y uso de las TIC (P.1.), competencia digital (P.2.), y actitudes y expectativas hacia las TIC (P.3.), se aplicaron estadísticos descriptivos, mientras que para la pregunta cuatro, sobre la posible existencia de diferencias generacionales entre ambos grupos, se aplicó la prueba no paramétrica $U$ de MannWhitney.

Tabla 2. Análisis de datos y preguntas de investigación

\begin{tabular}{|c|c|}
\hline $\begin{array}{l}\text { P.1. Disponibilidad de recursos digitales y uso de } \\
\text { las TIC }\end{array}$ & Estadísticos descriptivos \\
\hline P.2. Competencia digital & Estadísticos descriptivos \\
\hline P.3. Actitudes y expectativas hacia las TIC & Estadísticos descriptivos \\
\hline P.4. Diferencias generacionales & U de Mann-Whitney \\
\hline
\end{tabular}

Asimismo, se realizó una regresión entre las expectativas y actitudes hacia las TIC (P.3.) y la competencia digital del estudiantado (P.2.), pero no se halló relación entre ambas.

\section{RESULTADOS}

A continuación, se exponen los resultados derivados del análisis de datos divididos en dos bloques. En el primer bloque se describe el perfil global de los estudiantes que han participado en el estudio. Se exponen los resultados obtenidos en el cuestionario en relación a la autopercepción de la competencia digital y finalmente se detallan las expectativas y actitudes hacia las TIC. En el segundo bloque se comparan las medias de los dos grupos analizados, correspondientes a los dos planes de estudios.

\subsection{Disponibilidad de recursos digitales y uso de las TIC}

En cuanto al perfil de los estudiantes, como se puede observar en la tabla 3, las tecnologías que con mayor frecuencia están presentes en el hogar de los participantes son los dispositivos móviles $(99,33 \%)$ y la televisión $(98,32 \%)$, seguidas del ordenador portátil $(97,99 \%)$ en detrimento del ordenador de sobremesa $(45,97 \%)$, siendo esta la tecnología que menos está presente.

Tabla 3. Disponibilidad en el domicilio del estudiante

\begin{tabular}{lc}
\hline & Frecuencia (\%) \\
\hline 1. Ordenador de sobremesa & 45,97 \\
2. Ordenador portátil & 97,99 \\
3. Tableta & 63,09 \\
4. Móvil & 99,33 \\
5. Televisión & 98,32 \\
6. Videoconsola & 60,40 \\
7. Cámara de fotos & 64,43 \\
\hline
\end{tabular}

Respecto al empleo de los recursos tecnológicos en actividades relacionadas con el aprendizaje (tabla 4), en el ámbito universitario las dos herramientas que más se emplean son el ordenador portátil $(93,62 \%)$ y el móvil $(90,60 \%)$ mientras que los equipos informáticos facilitados por la propia institución solo se utilizan por el $28,86 \%$ y el uso de tableta por un $25,50 \%$. 
El uso educativo de la tecnología en el hogar se da preferentemente a través del ordenador portátil $(94,97 \%)$ y del dispositivo móvil $(82,89 \%)$ y en menor frecuencia, a través de tableta $(37,25 \%)$ y de ordenador de sobremesa $(20,47 \%)$.

Tabla 4. Uso de los recursos TIC en actividades de aprendizaje

\begin{tabular}{lc}
\hline & Frecuencia (\%) \\
\hline Uso educativo en la universidad & \\
1. Ordenador portátil & 93,62 \\
2. Tableta & 25,50 \\
3. Móvil & 90,60 \\
4. Equipos de la universidad & 28,32 \\
Uso educativo en el hogar & \\
5. Ordenador de sobremesa & 20,47 \\
6. Ordenador portátil & 94,97 \\
7. Tableta & 37,25 \\
8. Móvil & 82,89 \\
\hline
\end{tabular}

Al analizar el tiempo de uso tanto de los dispositivos, como de los recursos y aplicaciones (tabla 5), encontramos que el dispositivo más utilizado es el móvil $(4,72)$, seguido del ordenador portátil $(3,97)$ mientras que los menos utilizados derían el ordenador de sobremesa $(1,32)$ y la tableta $(1,89)$.

Las redes sociales son el recurso que durante más tiempo utilizan $(3,58)$, seguido del correo electrónico $(3,18)$ y de la mensajería instantánea $(2,93)$.

En cuanto a las aplicaciones, la plataforma educativa de la universidad es la más empleada $(3,21)$ seguida del trabajo colaborativo $(2,40)$, juegos $(2,01)$ y sistemas de videoconferencia $(1,89)$. Destaca la poca frecuencia de uso de aplicaciones como editores de sonido, vídeo e imagen (1,25, 1,48 y 1,86 respectivamente).

Tabla 5. Uso de los diferentes dispositivos, recursos y aplicaciones

\begin{tabular}{ll}
\hline & M (SD) \\
\hline Tiempo de uso de dispositivos & \\
1. Ordenador de sobremesa & $1,32(0,651)$ \\
2. Ordenador portátil & $3,97(1,075)$ \\
3. Tableta & $1,89(1,204)$ \\
4. Móvil & $4,72(0,638)$ \\
Tiempo de uso de recursos & \\
5. Correo electrónico & $3,18(1,425)$ \\
6. Mensajería instantánea & $2,93(1,595)$ \\
7. Redes sociales & $3,58(1,622)$ \\
Tiempo de uso de aplicaciones & \\
8. Editores de sonido & $1,25(0,555)$ \\
9. Editores de vídeo & $1,48(0,727)$ \\
10. Editores de imagen & $1,86(0,879)$ \\
11. Herramientas de trabajo colaborativo & $2,40(1,160)$ \\
12. Plataforma educativa de la universidad & $3,21(1,088)$ \\
13. Sistemas de vídeoconferencia & $1,89(1,156)$ \\
14. Juegos & $2,01(1,148)$ \\
\hline
\end{tabular}

Finalmente, en la tabla 6 se identifica la finalidad con la que los estudiantes hacen uso de las TIC. Al respecto se observa que el uso más elevado se da para conectar en redes sociales $(4,32)$ y comunicarse sincrónicamente $(4,15)$. También se utiliza con frecuencia para el entretenimiento $(4,01)$ y para el trabajo $(3,93)$. De uso más moderado se encuentra la formación $(3,89)$, compartir archivos $(3,88)$ e informarse $(3,55)$ o comunicarse asíncronamente $(3,37)$ mientras que con menor finalidad se utilizan para la gestión de la agenda $(2,87)$. 
Tabla 6. Finalidad de uso diario de las TIC

\begin{tabular}{lc}
\hline & $\mathbf{M}($ SD) \\
\hline 1. Entretenerse & $4,01(0,895)$ \\
2. Formarse & $3,89(0,755)$ \\
3. Trabajar & $3,93(0,966)$ \\
4. Informarse & $3,55(0,987)$ \\
5. Comunicarse síncronamente & $4,15(1,022)$ \\
6. Comunicarse asíncronamente & $3,37(1,056)$ \\
7. Compartir archivos & $3,88(0,970)$ \\
8. Conectarse en redes sociales & $4,32(0,939)$ \\
9. Gestionar/administrar agenda & $2,87(1,199)$ \\
10. Otras actividades & $2,86(1,116)$ \\
\hline
\end{tabular}

\subsection{La competencia digital}

En cuanto a los resultados de la prueba INCOTIC para la autopercepción de la competencia digital, tal y como se muestra en la tabla 7 , los usuarios se perciben con un nivel medio en competencia digital general $(3,58)$, siendo la alfabetización comunicativa la que obtiene mayor puntuación $(3,86)$, seguida de la alfabetización multimedia $(3,75)$, la alfabetización informacional $(3,46)$ y en último lugar la alfabetización tecnológica $(3,27)$.

No obstante, en los ítems de cada alfabetización se hallan puntuaciones individuales diversas. En la alfabetización comunicativa, destacan las puntuaciones en trabajo colaborativo $(4,73)$ y comunicación síncrona $(4,37)$ mientras que los valores inferiores se obtienen en la presentación de información $(2,80)$. En la alfabetización multimedia resaltan los valores para producir contenidos propios $(4,33)$ y para valorar el estilo de mensajes $(4,21)$ mientras que la menor puntuación se obtiene en la elección de medios apropiados $(2,62)$. En alfabetización tecnológica el ítem sobre configuración de dispositivos obtiene la puntuación más alta $(4,52)$ y el uso de internet la más baja $(2,27)$ y finalmente en la alfabetización informacional, el reconocimiento de la necesidad de información es la puntuación más elevada $(3,96)$ mientras que en la organización de la información se obtiene la menor puntuación $(2,88)$.

Tabla 7. Resultados descriptivos de la competencia digital y sus alfabetizaciones

\begin{tabular}{lc}
\hline & M (SD) \\
\hline Alfabetización informacional & $3,46(0,737)$ \\
1. Reconocer la necesidad de información & $3,96(0,746)$ \\
2. Localizar la información & $3,29(1,191)$ \\
3. Evaluar la información & $3,56(1,056)$ \\
4. Organizar la información & $2,88(1,189)$ \\
5. Transformar la información & $3,56(1,189)$ \\
Alfabetización tecnológica & $3,27(0,815)$ \\
6. Configurar dispositivos & $4,52(0,775)$ \\
7. Configurar aplicaciones & $2,67(1,298)$ \\
8. Usar herramientas de ofimática & $2,92(1,226)$ \\
9. Usar internet & $2,27(1,248)$ \\
10. Usar aplicaciones específicas & $3,70(1,168)$ \\
Alfabetización multimedia & $3,75(0,604)$ \\
11. Comprender mensajes & $3,60(0,884)$ \\
12. Valorar el estilo de mensajes & $4,21(0,733)$ \\
13. Analizar críticamente mensajes & $3,90(0,899)$ \\
14. Elegir los medios apropiados & $2,62(1,205)$ \\
15. Producir contenidos propios & $4,33(0,957)$ \\
Alfabetización comunicativa & $3,86(0,690)$ \\
16. Presentación & $2,80(1,161)$ \\
17. Comunicación asíncrona & $3,56(1,177)$ \\
18. Comunicación síncrona & $4,37(1,015)$ \\
\hline
\end{tabular}




\begin{tabular}{ll}
\hline 19. Publicación & $3,86(1,209)$ \\
20. Trabajo colaborativo & $4,73(0,674)$ \\
Competencia digital & $3,58(0,557)$ \\
\hline
\end{tabular}

\subsection{Actitudes y expectativas hacia las TIC}

En cuanto a las expectativas de uso, como se observa en la tabla 8, la puntuación media se sitúa en un 4,14, destacando la puntuación de los ítems relacionados con la comunicación $(4,61)$, con la autonomía $(4,41)$ y con la mejora de la docencia $(4,30)$ y obteniendo menor puntuación en los ítems relacionados con que las TIC favorecen la escritura $(3,36)$ o que deben estar más integradas en el plan de estudios $(4,04)$.

Sobre las actitudes hacia el uso de la tecnología, la puntuación media obtenida globalmente es de 3,95. En particular, las puntuaciones más altas las obtienen los ítems de buscadores web $(4,76)$, navegadores web $(4,72)$, presentaciones $(4,69)$ y plataforma educativa de la universidad $(4,65)$. Por el contrario, las puntuaciones más bajas se dan en juegos $(2,73)$, editores de sonido $(3,03)$, hojas de cálculo $(3,20)$ y gestores de correo $(3,30)$.

Tabla 8. Expectativas y actitudes hacia el uso de las TIC

\begin{tabular}{lc}
\hline & M (SD) \\
\hline Expectativas del uso de las TIC & $4,14(0,547)$ \\
1. Facilidad de uso & $4,12(0,740)$ \\
2. Sensación de disfrute & $4,20(0,766)$ \\
3. Autonomía & $4,41(0,632)$ \\
4. Comunicación & $4,61(0,605)$ \\
5. Mejora del aprendizaje & $4,05(0,829)$ \\
6. Motivación & $4,08(0,859)$ \\
7. Creatividad & $4,18(0,839)$ \\
8. Escritura & $3,36(1,098)$ \\
9. Integración en el plan de estudios & $4,04(0,854)$ \\
10. Mejora de la docencia & $4,30(0,772)$ \\
Actitudes hacia el uso de herramientas & $3,95(0,523)$ \\
11. Procesador de textos & $4,57(0,746)$ \\
12. Bases de datos & $3,78(1,100)$ \\
13. Hojas de cálculo & $3,20(1,184)$ \\
14. Presentaciones & $4,69(0,558)$ \\
15. Editores de sonido & $3,03(1,090)$ \\
16. Editores de vídeo & $3,50(0,974)$ \\
17. Editores de imagen & $3,46(0,988)$ \\
18. Gestores de correo & $4,30(0,898)$ \\
19. Navegadores web & $4,72(0,603)$ \\
20. Buscadores web & $4,76(0,548)$ \\
21. Editores de blog/web & $3,86(0,907)$ \\
22. Herramientas de trabajo colaborativo & $4,54(0,775)$ \\
23. Plataforma educativa de la universidad & $4,65(0,658)$ \\
24. Mensajería instantánea & $4,27(0,948)$ \\
25. Sistema de videoconferencia & $3,67(1,240)$ \\
26. Redes sociales & $3,82(1,081)$ \\
27. Juegos & $2,73(1,234)$ \\
\hline
\end{tabular}

Si bien no era el objetivo principal de la presente investigación, se ha llevado a cabo una regresión con el objeto de comprobar si existe relación entre las expectativas y actitudes hacia las TIC y la CD, similar a la realizada por González et al. (2018). Al igual que para dichos autores, tras un análisis preliminar, los resultados han sido negativos, no se ha encontrado relación entre ambos elementos. 


\subsection{Comparación de medias entre distintos planes de estudio}

Al comparar las medias de las alfabetizaciones digitales en los dos planes de estudio (tabla 9) se encuentra que los alumnos del plan de 2018 obtienen mejores puntuaciones que los del plan de 2010 en la competencia digital general $(3,60$ frente a 3,56) y en la alfabetización comunicativa $(4,03$ frente a 3,68), mientras que el alumnado del plan de 2010 supera al del 2018 en las alfabetizaciones de la competencia digital informacional $(3,48$ frente a 3,44$)$, tecnológica $(3,32$ frente a 3,22$)$ y multimedia $(3,77$ frente a 3,72$)$.

\begin{tabular}{|c|c|c|}
\hline & $\begin{array}{c}\text { Plan } 2010 \\
\text { M (SD) }\end{array}$ & $\begin{array}{c}\text { Plan } 2018 \\
\text { M (SD) }\end{array}$ \\
\hline Alfabetización informacional & $3,48(0,77)$ & $3,44(0,71)$ \\
\hline Alfabetización tecnológica & $3,32(0,83)$ & $3,22(0,80)$ \\
\hline Alfabetización multimedia & $3,77(0,66)$ & $3,72(0,55)$ \\
\hline Alfabetización comunicativa & $3,68(0,73)$ & $4,03(0,60)$ \\
\hline Competencia digital & $3,56(0,61)$ & $3,60(0,50)$ \\
\hline Expectativas de uso de TIC & $4,01(0,56)$ & $4,27(0,51)$ \\
\hline Actitudes hacia el uso de herramientas & $3,86(0,51)$ & $4,03(0,52)$ \\
\hline
\end{tabular}

Por otro lado, el alumnado del nuevo plan obtiene un resultado más alto tanto en expectativas sobre el uso de las TIC $(4,27$ frente a 4,01$)$ como en actitud hacia el uso de herramientas tecnológicas $(4,03$ frente a 3,86$)$.

En la figura 1, se puede observar más claramente como, tanto en la competencia digital general como en alfabetización comunicativa y tanto en expectativas, como en las actitudes hacia las herramientas TIC se obtuvo una mayor puntuación por parte de los estudiantes del plan nuevo.

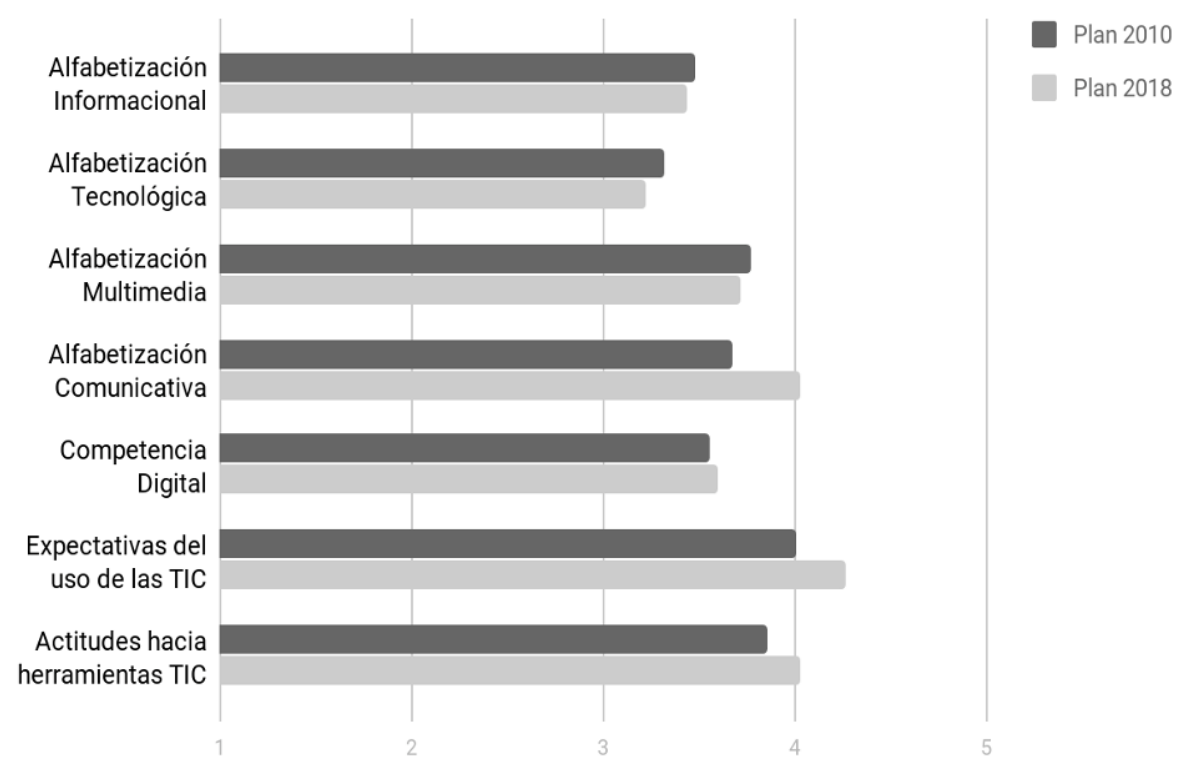

Figura 1. Puntuaciones medias de las diferentes variables según el plan de estudios 
Para analizar tales diferencias, y tras comprobar que las variables no cumplen el principio de normalidad, se realizó la prueba no paramétrica $U$ de Mann-Whitney.

Tabla 10. Comparación de medias según la prueba U de Mann-Whitney

\begin{tabular}{lcccc}
\hline & $\begin{array}{c}\text { U de Mann- } \\
\text { Whitney }\end{array}$ & Z & Sig. (bilateral) & r (effect size) \\
\hline A. Informacional & 10569.5 & -0.510 & 0.610 & 0.03 \\
A. Tecnológica & 10159.5 & -1.068 & 0.285 & 0.06 \\
A. Multimedia & 10558.0 & -0.527 & 0.598 & 0.03 \\
A. Comunicativa & 7853.5 & -4.217 & $0.000^{*}$ & 0.25 \\
$\quad$ Competencia digital & 10669.5 & -0.373 & 0.709 & 0.02 \\
Expectativas & 7883.5 & -4.165 & $0.000^{*}$ & 0.24 \\
Actitudes & 8909.5 & -2.765 & $0.006^{*}$ & 0.16 \\
\hline
\end{tabular}

Como se puede observar en la tabla10, a pesar de que no existen diferencias significativas en la competencia digital general, sí se detectaron diferencias en una de las áreas que la conforman, la alfabetización comunicativa $(p=0.000)$. Del mismo modo, también resultaron significativas las diferencias en las variables expectativas $(p=0.000)$ y actitudes $(p=0.006)$.

\section{DISCUSIÓN}

El presente trabajo se ha centrado en explorar el perfil digital de los estudiantes universitarios actuales. Un perfil digital compuesto por el uso que hacen de la tecnología, la percepción sobre su propia competencia digital, y las actitudes y expectativas que tienen hacia el uso de las TIC. A continuación se discuten los resultados obtenidos dando respuesta a las preguntas de investigación planteadas en la introducción.

En relación con la primera pregunta, acerca de las características digitales de los estudiantes y de la disponibilidad y uso de la tecnología, se ha evidenciado el marcado carácter digital de los estudiantes. Como apunta Livingstone, et al. (2019), los estudiantes tienen cada vez más dispositivos móviles, y la mayor parte de ellos disponen también de ordenador portátil y televisión en su hogar, y en menor medida, cámaras fotográficas, tabletas o videoconsolas. Respecto al uso que hacen de la tecnología, la mayor parte de los participantes en este estudio utilizan el ordenador portátil y el móvil como herramientas principales de trabajo, tanto en la universidad como en sus hogares, resultados similares a los obtenidos por Camacho y Esteve (2018), y Goggin (2013). Estos dispositivos son principalmente empleados para consultar la plataforma educativa de la universidad y para trabajar con herramientas colaborativas, datos que contrastan en cambio con los presentados por Stoilova et al. (2020), quienes obtuvieron un uso principalmente de carácter lúdico.

En cuanto a la segunda pregunta de investigación, en referencia a la competencia digital, los estudiantes actuales se perciben a sí mismos con una competencia digital elevada, hecho que a priori podría denotar que están preparados para enfrentarse a los retos de la sociedad actual, así como para desempeñar su futura profesión docente en un mundo cada vez más digital (Caena \& Redecker, 2019; Castañeda et al., 2018). Los resultados más elevados se obtienen en las alfabetizaciones comunicativa y multimedia, en la línea de las características atribuidas a esta generación digital, como el uso frecuente de redes sociales, en las cuales tiene gran importancia la imagen y el contenido audiovisual (Boyd, 2014). En este sentido, destaca la alta capacidad con la que se perciben para trabajar de manera síncrona y colaborativa, así como para producir y consumir contenidos audiovisuales. En contraste, llama la atención, el poco tiempo que dedican al uso de editores de vídeo, sonido e imagen.

Respecto a la tercera cuestión, sobre las actitudes y expectativas hacia el uso de las TIC en su futura vida profesional, parece que son muy elevadas, e incluso superiores a sus propios niveles de competencia digital. Estos resultados sugieren que las tecnologías les resultan de utilidad en su contexto laboral y personal, y que están alineadas con sus propios valores y experiencias previas. Como plantean Windschitl y Sahl (2002), las actitudes hacia las TIC, así como el conocimiento de estas y la propia competencia digital, son elementos predictores del uso de la tecnología en el aula. A la luz de estos resultados, cabe esperar que los alumnos participantes utilicen la tecnología en su futuro profesional. Sin embargo, como apuntan Guo et 
al. (2013), será determinante que cuenten con referentes y modelos de profesionales que utilizan las tecnologías digitales cuando realicen prácticas en centros educativos.

Finalmente, para responder a la última pregunta de investigación, se comprobaron las posibles diferencias existentes entre los estudiantes que respondieron al cuestionario en dos momentos temporales y dos planes de estudios diferentes. En este sentido, se evidencian diferencias significativas a favor de los estudiantes del nuevo plan de estudios, en relación con la dimensión comunicativa de la competencia digital, así como de las actitudes y expectativas hacia las TIC, tanto hacia su uso en el ámbito doméstico como en el ámbito educativo. Estos datos, al igual que sugieren Kirschner \& De Bruyckere (2017), se deben tomar con cierta cautela, no se puede afirmar con rotundidad que las generaciones actuales sean digitalmente competentes en comparación con generaciones anteriores. Tampoco se puede afirmar de manera categórica que la intensificación de la formación práctica propia del plan nuevo haya influido en el resultado, para ello, el estudio deberá replicarse en el futuro.

\subsection{Conclusiones}

En esta investigación se explora la competencia digital de los estudiantes que cursan el Grado de Maestro en Educación Primaria en dos planes de estudio diferentes, así como sus expectativas y actitudes hacia el uso de las tecnologías digitales en su vida cotidiana y en el ámbito académico.

Los resultados nos permiten obtener una visión actualizada del estado de la cuestión, mostrando una buena experiencia y un uso frecuente de la tecnología por parte de los jóvenes, en combinación con una actitud de apertura hacia la integración de las TD en su futura docencia.

En los planes de estudio de los grados de maestro, se siguen detectando carencias formativas en áreas específicas de la competencia digital, como la alfabetización informacional, relacionada con la búsqueda, selección y organización de la información o la alfabetización tecnológica, vinculada al adecuado uso de la red y a la configuración de dispositivos. Ambas alfabetizaciones resultarán de especial relevancia profesional para las nuevas generaciones de docentes.

Los resultados también muestran una imagen de los recursos educativos más necesarios para el alumnado actual. A este respecto, las instituciones universitarias ya no deben invertir tanto en proporcionar dispositivos informáticos, sino en facilitar el acceso a internet, así como a herramientas y aplicaciones específicas para realizar las tareas académicas.

Por otro lado, al comparar los resultados obtenidos por los estudiantes del plan de estudios de 2010 con los del plan de estudios de 2018, encontramos que la percepción sobre la competencia digital de unos y otros no se ha modificado sustancialmente durante estos años. En este sentido, se podría concluir que la formación previa realizada en anteriores niveles educativos o la modificación del plan de estudios, no han incidido significativamente en la mejora de la competencia digital. Las diferencias se centran especialmente en el ámbito comunicativo de esta competencia, algo que parece estar ligado a los hábitos de uso que tienen los jóvenes hoy en día.

Entre las limitaciones de este estudio, cabe mencionar el hecho de que la muestra no es lo suficientemente amplia como para poder generalizar los resultados obtenidos. En investigaciones futuras, se pretende ampliar dicha muestra con este fin.

Finalmente, señalar que este trabajo concluyó durante la primera semana del confinamiento por la COVID-19 en España. Dicho confinamiento impuso a la población la necesidad de utilizar diversas herramientas de comunicación síncrona y asíncrona para mantener el contacto con la red socio-familiar, y en el caso del estudiantado, para continuar con su formación académica. Siendo conscientes de que la propia situación de confinamiento, por lo excepcional, puede haber influido los resultados obtenidos, se pretende por ello, seguir profundizando durante los próximos cursos, con el fin de afianzar la tendencia de dichos resultados. Por otro lado, no es menos cierto que los hechos sucedidos a raíz de la pandemia han abierto una serie de caminos a explorar, en relación con el empleo de la tecnología en educación y a la percepción de la competencia digital de todos los miembros de la comunidad educativa. 


\section{ENLACES}

Cuestionario en línea: https://cutt.ly/mxtziWk

\section{REFERENCIAS BIBLIOGRÁFICAS}

Ala-Mutka, K. (2011). Mapping digital competence: Towards a conceptual understanding. Institute for Prospective Technological Studies. https://bit.ly/3qYETP8

Anderson, M. \& Jiang, J. (2018). Teens' social media habits and experiences. Pew Research Center, 28. https://bit.ly/3c1ybnn

Antunovic, D., Parsons, P. \& Cooke, T. R. (2018). 'Checking' and googling: Stages of news consumption among young adults. Journalism, 19(5), 632-648. https://doi.org/10.1177/1464884916663625

Bawden, D. (2008). Origins and concepts of digital literacy. Digital literacies: Concepts, policies and practices, 30, 17-32.

Boyd, D. (2014). It's complicated: The social lives of networked teens. Yale University Press.

Bullen, M., Morgan, T. \& Qayyum, A. (2011). Digital Learners in Higher Education: Generation is Not the Issue. Canadian Journal of Learning and Technology, 37(1). https://doi.org/10.1111/ejed.12345

Caena, F. \& Redecker, C. (2019). Aligning teacher competence frameworks to 21st century challenges: The case for the European Digital Competence Framework for Educators ( Digcompedu). European Journal of Education, 54(3). https://doi.org/10.1111/ejed.12345

Calvani, A., Fini, A., Ranieri,M. \& Picci, P.(2012). Are young generations in secondary school digitally competent? A study on Italian teenagers. Computers \& Education, 58(2), 797- 807. https://doi.org/10.1016/j.compedu.2011.10.004

Camacho, M. \& Esteve, F. M. (2018). El uso de las tabletas y su impacto en el aprendizaje. Una investigación nacional en centros de Educación Primaria. Revista de Educación, 379. https://doi.org/10.4438/1988-592X-RE-2017-379-366

Claro, M., Preiss, D. D., Martín, E. S., Jara, I., Hinostroza, J. E., Valenzuela, S., Cortes, F. \& Nussbaum, M. (2012). Assessment of 21st century ICT skills in Chile: Test design and results from high school level students. Computers \& Education, 59(3), 1042-1053. https://doi.org/10.1016/i.compedu.2012.04.004

Comisión Europea. (2018). Recomendación del Consejo de 22 de mayo de 2018 relativa a las competencias clave para el aprendizaje permanente. Diario Oficial de la Unión Europea, 46-2018, C 189, 1-13. https://bit.ly/3rV6AJU

Covello, S. \& Ley, J. (2010). A review of Digital Literacy Assessment Instruments. Syracuse University, 1-31. https://bit.ly/3bUeoWO

Davis, F. (1989). Perceived Usefulness, Perceived Ease of Use and User Acceptance of Information Technology. Quarterly MIS, 319-340. https://doi.org/10.2307/249008

Domingo, M. \& Marquès, P. (2011). Aulas 2.0 y uso de las TIC en la práctica docente. Comunicar, 19(37), 169-175. https://bit.ly/2OlqJV7

Ferrari, A., Punie, Y. \& Redecker, C. (2012). Understanding digital competence in the 21st century: An analysis of current frameworks. In A. Ravenscroft, S. Lindstaedt, C. Delgado y D. Hernández-Leo (Eds.), 21st century learning for 21st century skills, (pp. 79-92). Springer. https://doi.org/10.1007/978-3-642-33263-0 7

Ferrari, A. (2013). DIGCOMP: A framework for developing and understanding digital competence in Europe. Institute for Prospective Technological Studies (IPTS), European Commission. https://bit.ly/3cla91G 
Gallardo-Echenique, E. E., Marqués-Molías, L., Bullen, M., \& Strijbos, J. W. (2015). Let's talk about digital learners in the digital era. International Review of Research in Open and Distributed Learning, 16(3), 156-187. https://doi.org/10.19173/irrodl.v16i3.2196

Gilster, P. (1997). Digital literacy. Wiley Computer.

Goggin, G. (2013). Youth culture and mobiles. Mobile Media \& Communication, 1(1), 83-88. https://doi.org/10.1177/2050157912464489

González, J., Esteve-Mon, F. M., Larraz, V., Espuny, C., \& Gisbert, M. (2018). INCOTIC 2.0. Una nueva herramienta para la autoevaluación de la competencia digital del alumnado universitario. Profesorado: Revista de curriculum y formación del profesorado, 22(4), 133152. https://doi.org/10.30827/profesorado.v22i4.8401

Guo, X., Sun, Y., Wang, N., Peng, Z. \& Yan,Z. (2013). The dark side of elderly acceptance of preventive mobile health services in China. Electronic Markets, 23(1), 49-61. https://bit.ly/3cCwBr8

Hatlevik, O. E. \& Christophersen, K.-A. (2013). Digital competence at the beginning of upper secondary school: Identifying factors explaining digital inclusion. Computers \& Education, 63, 240-247. https://doi.org/10.1016/i.compedu.2012.11.015

Karahanna, E., Agarwal, R. \& Angst, C. M. (2006). Reconceptualizing compatibility beliefs in technology acceptance research. MIS quarterly, 30(4), 781-804. https://doi.org/10.2307/25148754

Kennedy, G., Dalgarno, B., Gray, K., Judd, T., Waycott, J., Bennett, S., Maton, K., Krause, K. L., Bishop, A. \& Chang, R. (2007). The net generation are not big users of Web 2.0 technologies: Preliminary findings. ICT: Providing choices for learners and learning, 517-525. https://bit.ly/3tr4SAj

Kirschner, P. A. \& De Bruyckere, P. (2017). The myths of the digital native and the multitasker. Teaching and Teacher Education, 67, 135-142. https://doi.org/10.1016/i.tate.2017.06.001

Larraz, V. (2012). La competència digital a la Universitat. [Tesis doctoral]. Universitat d'Andorra.

Livingstone, S.; Kardefelt, D. \& Saeed, M. (2019). Global Kids Online Comparative Report, Innocenti Research Report. UNICEF Office of Research. https://bit.ly/3eJgRFd

Martin, A. (2005). DigEuLit-a European framework for digital literacy: A Progress Report. Journal of eLiteracy, 2(2), 130-136.

Meyers, E. M., Erickson, I. \& Small, R. V. (2013). Digital literacy and informal learning environments: An introduction. Learning, Media and Technology, 38(4), 355-367. https://doi.org/10.1080/17439884.2013.783597

Oblinger, D. G. \& Oblinger, J. L. (2005). Educating the net generation. Educause.

Oreg, S. (2003). Resistance to change: Developing an individual differences measure. Journal of applied psychology, 88(4), 680. https://doi.org/10.1037/0021-9010.88.4.680

Pedró, F. (Septiembre de 2009). New Millennium learners in higher education: Evidence and policy implications. [Sesión de conferencia] International Conference on 21st Century Competencies, Centre for Educational Research and Innovation (CERI), Bruselas. https://bit.ly/3vHkZvq

Prendes, M. P., Castañeda, L. \& Gutiérrez, I. (2010). Competencias para el uso de TIC de los futuros maestros. Comunicar, Revista Científica de Educomunicación, 18(35). https://bit.ly/2P3p9gg

Prensky, M. (2001). Digital Natives, Digital Immigrants. On the Horizon, 9(5), 1-6. https://doi.org/10.1108/10748120110424816

Rogers, E. M. (1962). Diffusion of innovations. Free Press of Glencoe. 
Selwyn, N. (2009). The digital native-myth and reality. Aslib Proceedings: New Information Perspectives, 61(4). https://doi.org/10.1108/00012530910973776

Siddiq, F. \& Scherer, R. (2019). Is there a gender gap? A meta-analysis of the gender differences in students' ICT literacy. Educational Research Review, 27, 205-217. https://doi.org/10.1016/i.edurev.2019.03.007

Solomon, A., Wilson, G. \& Taylor, T. (2011). 100\% Information Literacy Success. Nelson Education.

Spante, M., Hashemi, S. S., Lundin, M. \& Algers, A. (2018). Digital competence and digital literacy in higher education research: Systematic review of concept use. Cogent Education, 5(1), 1-21. https://doi.org/10.1080/2331186X.2018.1519143

Stoilova, M., Livingstone, S. \& Mascheroni, G. (2020). Digital childhood? Global perspectives on children and mobile technologies. In R. Ling, L. Fortunati, G. Gog - gin, Y. Li \& S. S. Lim (Eds.), The Oxford Handbook of Mobile Communication and Society (pp. 129-143). Oxford University Press.

Tejedor, F.J. \& García-Varcárcel, A. (2006). Competencias de los profesores para el uso de las TIC en la enseñanza. Análisis de sus conocimientos y actitudes. Revista Española de Pedagogía, 233; 21-44. https://bit.ly/30QBjfe

Valtonen, T., Pontinen, S., Kukkonen, J., Patrick, D., Väisänen, P. \& Hacklin, S. (2011). Confronting the technological pedagogical knowledge of Finnish Net Generation student teachers. Technology, Pedagogy and Education, 20(1), 3-18. https://doi.org/10.1080/1475939X.2010.534867

Venkatesh, V. \& Bala, H. (2008). Technology acceptance model 3 and a research agenda on interventions. Decision sciences, 39(2), 273-315. https://doi.org/10.1111/j.15405915.2008.00192.x

Vogels, E., Perrin, A., Rainie, L. \& Anderson, M. (2020). 53\% of Americans Say the Internet Has Been Essential during the COVID-19 Outbreak. Pew Research Center. https://pewrsr.ch/312olFL

Windschitl, M. \& Sahl, K. (2002). Tracing teachers' use of technology in a laptop computer school: The interplay of teacher beliefs, social dynamics, and institutional culture. American educational research journal, 39(1), 165-205. https://doi.org/10.3102/00028312039001165 


\section{INFORMACIÓN SOBRE LOS AUTORES}

\section{María Ángeles Llopis Nebot}

Universitat Jaume I de Castellón

Es profesora asociada del área de Didáctica y Organización escolar en el Departamento de Pedagogía de la Universitat Jaume I. Centra su docencia en los grados de Educación Primaria e Infantil. Doctora en Educación por la misma universidad, y es miembro del grupo de investigación GREAT (Enseñanza, Aprendizaje y Tecnología). Su investigación se centra en el pensamiento reflexivo y computacional, y en la competencia digital.

\section{María Santágueda Villanueva}

Universitat Jaume I de Castellón

Es profesora ayudante doctor en el Departamento de Educación y Didácticas Específicas de la Universitat Jaume I, impartiendo didáctica de las matemáticas en los grados de Educación Primaria e Infantil. Doctora en Matemática Aplicada por la Universidad de Valencia, participa en los grupos de investigación ENDAVANT y DIMATHEX, centrando su investigación en didáctica de las matemáticas, neurociencia y la atención a la diversidad.

\section{Francesc Marc Esteve Mon}

Universitat Jaume I de Castellón

Es profesor Contratado Doctor del área de Didáctica y Organización Escolar, en el Departamento de Pedagogía de la Universitat Jaume I. Doctor internacional en Tecnología Educativa por la Universitat Rovira i Virgili, y miembro del grupo de investigación GREAT (Enseñanza, Aprendizaje y Tecnología), centra su investigación en el análisis de la competencia digital y la formación docente, así como en el estudio de la metodología de investigación Design-Based Research.$$
\text { (c) (1) (9) }
$$

Los textos publicados en esta revista están sujetos a una licencia de Reconocimiento 4.0 España de Creative Commons. Puede copiarlos, distribuirlos, comunicarlos públicamente y hacer obras derivadas siempre que reconozca los créditos de las obras (autoría, nombre de la revista, institución editora) de la manera especificada por los autores o por la revista. La licencia completa se puede consultar en:Licencia Creative Commons Atribución-NoComercial-Compartir por igual 4.0 Internacional.
} 